\title{
Aporte metodológico de la educación física, fortaleciendo la lectoescritura en los estudiantes de segundo EGB.
}

Methodological contribution of physical education, strengthening literacy in second EGB students.

Lic. Zoila Eugenia Guerrero Ávila Mgtr. ${ }^{1}$, Lcda. Luisa Valeria Guevara Flores MSc. ${ }^{2}$ \& Lic. Yury Douglas Barrios Palacios, $\mathrm{MSc}^{3}$.

DOI: https://doi.org/10.33262/concienciadigital.v3i4.1437

\section{Resumen}

Cuando un ciudadano ecuatoriano puede recordar a sus profesores de la escuela, por nombre, es porque ellos hicieron un gran aporte a su formación, como estudiante, alfabetizándolo. Es importante la enseñanza de la Lectoescritura en el segundo año e Educación Básica y que en esa etapa sea bien aprendida ya que de ahí se parte para los futuros aprendizajes de Lengua y Literatura que se desarrollaran durante toda la vida estudiantil, pero en esta ocasión se pretende motivar a los estudiantes a realizar un eficiente aprendizaje de manera recreativa fortalecida desde las clases de Educación física realizando un trabajo en conjunto con la docente del aula con el único fin que es permitirle al estudiante un aprendizaje integrador y motivador.

Palabras claves: Metodológico, Educación Física, fortaleciendo, lectoescritura, estudiantes, segundo EGB.

\section{Abstract.}

When an Ecuadorian citizen can remember his teachers from school, by name, it is because they made a great contribution to his training, as a student, alphabetizing him. It is important to teach Literacy in the second year and Basic Education and that at that

\footnotetext{
${ }^{1}$ Universidad de Guayaquil, Facultad de Educación Física Deportes y Recreación, zoila.guerreroa@ug.edu.ec

${ }^{2}$ Universidad de Guayaquil, Guayaquil- Ecuador, luisa.guevaraf@ug.edu.ec

${ }^{3}$ Universidad de Guayaquil, Guayaquil- Ecuador, yury.barriosp@ug.edu.ec
} 
stage it is well learned since from there we start for future language and Literature learning that will be developed throughout the student's life, but this time it is intended to motivate students to carry out an efficient learning in a recreational way strengthened from Physical Education classes by doing a job in conjunction with the classroom teacher with the sole purpose that is to allow the student an integrative and motivating learning.

Keywords: Learning, Creativity, Social Responsibility, Students, Strategies, Teaching Guide.

\section{Introducción}

La modernización en el mundo está reemplazando los intereses por la lectura en los estudiantes y se centra su atención hacia los videojuegos y la vida social virtual en todos los estratos de la sociedad, lo cual causa una problemática en nuestra juventud ya que vienen arrastrando ciertos vacíos académicos que de no ser atendidos a tiempo se transforman en un gran problema futuro. Esta problemática se puede contrastar haciendo la labor docente más divertida para los estudiantes en tal sentido se propone una enseñanza de la lectoescritura desde la óptica del docente de educación física en donde a nivel escolar se trabaja netamente recreativa despertando siempre en interés de cada uno de estudiantes en donde el lema principal será "aprendiendo jugando".

(Ferreriro \& Goméz Palacios, 2000) Refieren: "En las discusiones pedagógicas, el aprendizaje de la lectura y escritura ha sido tradicionalmente considerado como un proceso psicológico, un asunto de percepción e interpretación de símbolos gráficos”. pág. 207

(Bravo Valdivieso, 1990) Manifiesta "Las dificultades del aprendizaje constituye un severo $\mathrm{y}$ frecuente problema para muchos educadores y padres, cuyos niños no logran un rendimiento escolar acorde con sus expectativas o a nivel de los propios esfuerzos que hacen para aprender”. pag.15

En los primeros cursos de la educación básica y específicamente en el segundo año de educación general básica se presentan dificultades para el aprendizaje de los contenidos de lenguaje, de tal modo que se visualizan problemas específicos en la lectura y escritura, ambas actividades van concatenadas y se torna en la dificultad de lo que ahora conocemos como la lectoescritura, la misma que cuando se torna como una dificultad en el momento de su aprendizaje, la labor docente es investigar cuales son los factores que inciden en ese bloqueo que no permite una eficiente recepción de dichos conocimientos por parte de los estudiantes

y en ciertas ocasiones es el reflejo de traumas personales, problemas de atención, problemas interpersonales, etc., obstaculizando el aprendizaje de los estudiantes. 
(Veiga Alén \& Ideaspropias Editorial, 2005) expresan: "Cuando los niños tienen problemas de aprendizaje, estos suelen manifestarse mediante alguna característica especifica como por ejemplo dificultades al deletrear palabras, letra desordenada o sostener el lápiz torpemente, no comprender lo que lee, no recordar los sonidos de las letras o no comprender bromas o sarcasmos, etc." pág. 4

Por lo general en la escuela en sus primeros años es cuando el maestro suele detectar algún problema que pueda presentarse en el estudiante ya sea de carácter académico como conductual, y es ahí cuando el docente tiene que tener el criterio pedagógico para derivar dicho caso al DECE para que continúe el seguimiento y converse con los padres de familia sobre las pautas a seguir para la ayuda inmediata del estudiante.

(Duque Yepes, 2000) manifiesta "La pedagogía y la psicología han ido descubriendo la importancia del juego como medio de aprendizaje. Ahora es de común ocurrencia la utilización del juego para la enseñanza, desde el jardín hasta la alta gerencia. La recreación se convierte así en un instrumento pedagógico para transitar y producir actitudes”. pag. 55

(Coronado, 2002) Comenta "Uno de los problemas más arduos para iniciar a un alumno en la disciplina de la lectura es, sin duda, la motivación”. Pag.10

Se conoce que todo niño aprende jugando, que los contenidos académicos que son impartidos de manera recreativa son asimilados de manera eficiente por los estudiantes, por tal motivo se pretende realizar una unificación de contenidos, es decir, que los contenidos que la maestra de aula (profesora de educación básica) y ser fortalecidos en la clase de educación física (docente de educación física) de una forma recreativa, es decir con actividades lúdicas, para de tal forma propender al mejoramiento de la enseñanza aprendizaje de cada uno de los estudiantes del segundo año de educación básica los mismos que en su pensum académico inician con el trabajo fonológico específico para aprender a leer y escribir, que quiera o no es la base para toda la vida estudiantil.

\section{Desarrollo.}

Todo niño desde antes de llegar a la escuela ya conoce que la lectura y la escritura existen desde el momento que sus padres le leen un cuento, al observar en la calle ciertos rótulos, al ver frases en ciertos objetos en casa, etc., pero no conoce la técnica para el deletreo de cada palabra hasta después que llega a la escuela y de manera metodológica su maestra le enseña los rasgos principales para formar cada una de las letras así como el sonido especifico que tienen.

(Cuentos Vega, 2010) Manifiesta "Existe una gran variedad de métodos de enseñanza de la lectura, aunque todos pueden ser clasificados en dos categorías: los sintéticos y los analíticos". pag.189 
La lectoescritura es un proceso de aprendizaje que requiere de una metodología específica para su desarrollo, el docente en su afán de enseñar todas las estrategias necesarias para que sus estudiantes vayan aprendiendo todos los procesos fonológicos se dota de una serie de herramientas y técnicas metodológicas así como de métodos de enseñanza ya existentes tales como: el método alfabético, el método fonético, el método silábico, el método global, el método generadora y el método constructivista. El docente de aula escogerá el método que más domine para de tal manera ir desarrollando en sus estudiantes el conocimiento fonológico.

Las clases de educación física a nivel escolar son netamente recreativas, ya que se conoce por investigaciones anteriores que el niño aprende jugando y de esta premisa nos respaldamos para pretender la unificación de los aprendizajes, es decir lo que la maestra de lenguaje en el aula enseña será fortalecido en la clase de educación física, pero desde una óptica recreativa, utilizando como herramienta fundamental un área abierta como es el patio de la Institución Educativa.

(Libros, 1999) "Recrear es generar algo nuevo, ese otro modo de ser que pone al ser en libertad". Pag.23

(Educativas, 2004) "El juego y el aprendizaje han estado siempre en la esencia de todos los debates, discusiones y propuestas que giran alrededor de la educación infantil”. Pag.1

Con esta propuesta innovadora se propone conseguir que los estudiantes creen sus propias posibilidades de aprendizaje y a la vez se motiven a conocer y desarrollar la parte de la lectoescritura para obtener unos resultados favorables al termino del año lectivo lo cual servirá para toda la vida.

Para este proceso de unificación de metodologías de enseñanza se requiere que trabajen de manera conjunta la docente del aula con la maestra de educación física, en donde deberán programar constantes reuniones para delinear cada uno de los procesos de enseñanza aprendizaje que se están impartiendo con los estudiantes para el desarrollo de la lectoescritura.

Se deberá ir haciendo pruebas para medir constantemente el nivel de aprendizaje de los estudiantes del segundo año de educación básica, este proceso de evaluación será en ambos campos, tanto en el salón de clase como en las actividades recreativas que se realicen durante la clase de educación física.

Se conoce que para una educación integral se requiere de un grupo de docentes multidisciplinarios, esta propuesta pretende ser una de ellas en donde el beneficio en el rendimiento académico se va a ver reflejado en cada una de las mejoras y sobre todo en el 
éxito de cada uno de los participantes, en este caso cada uno de los estudiantes de segundos años de educación básica que son los agentes de estudio.

\section{Conclusiones.}

- Se torna indispensable el aprendizaje de la lectura y escritura en los primeros años de escolaridad y de estar bien segmentados dicho conocimiento se podría asegurar el éxito durante toda la etapa estudiantil, ya que de ahí se parte para el resto de los conocimientos.

- Esta propuesta se torna prometedora e innovadora ya que sera fortalecida desde el área de Educación Física, desde la óptica específicamente recreativa, por tal motivo los estudiantes se sentirán más motivados para realizar cada una de las actividades de manera eficiente en donde se conseguirá mayor atención de cada uno de los participantes para aprender todos los conocimientos que da la maestra en el aula, la misma que se tornara necesaria para luego completar cada una de las actividades recreativas de manera proactiva para causar el disfrute personal y el de todo el grupo humano que forman parte de los estudiantes del salón.

- Pero ningún proceso educativo seria eficaz si no se cuenta con la colaboración de los padres de familia los mismo que contribuirán para que sus hijos cumplan con sus tareas, fortalezcan sus conocimientos en casa, así como colaborar con cada uno de los materiales pedagógicos que se requieren para el desarrollo de cada clase tanto en el aula como en el patio de la Institución. Si los estudiantes se sienten apoyados se podría llegar a obtener todos los objetivos planteados antes durante y después de cada una de las clases, para llegar al objetivo principal que sería observado al término del año lectivo, donde los estudiantes lleguen a obtener una correcta lectura y escritura la misma que servirá para toda la vida.

\section{Referencias Bibliográficas.}

Coronado, J. (2002). Para leer mejor: Lecturas comentadas. Volumen 1. Mexico D.F.: Limusa.

Cuentos Vega, F. (2010). Psicología de la lectura. Madrid: WK Educación.

Ferreriro, E., \& Goméz Palacios, M. (2000). Nuevas perspectivas sobre los procesos de lectura y escritura. F.C. Mexico: Siglo XXI.

Bravo Valdivieso, L. (1990). Psicología de las dificultades del Aprendizaje. Santiago de Chile: Universitaria.

Duque Yepes, H. (2000). Pedagogía del tiempo libre. Santa Fe de Bogota: San Pablo.

Feldman J. (2005.). Autoestima ¿Cómo desarrollarla?: Juegos, actividades, recursos, experiencias. (Tercera ed.). Madrid, España: Narcia.

Veiga Alén, M., \& Ideaspropias Editorial. (2005). Dificultades de aprendizaje: Detección, prevensión y tratamiento. Ideaspropias Editorial S.L. 
Educativas, E. N. (2004). El Juego en la educación infantil: crecer jugando y aprendiendo. Buenos Aires: Noveduc Libros.

Libros, N. (1999). Recreación: Jugar y aprender cuando terminan las clases. Buenos Aires: Novedades Educativas.

Veiga Alén, M., \& Ideaspropias Editorial. (2005). Dificultades de aprendizaje: Detección, prevensión y tratamiento. Ideaspropias Editorial S.L. 
PARA CITAR EL ARTÍCULO INDEXADO.

Guerrero Ávila, Z. E., Guevara Flores, L. V., \& Barrios Palacios, Y. D. (2020). Aporte metodológico de la educación física, fortaleciendo la lectoescritura en los estudiantes de $\begin{array}{lllll}\text { segundo } & \text { EGB } & \text {. } & \text { ConcienciaDigital, } & 3(4),\end{array}$ https://doi.org/10.33262/concienciadigital.v3i4.1437

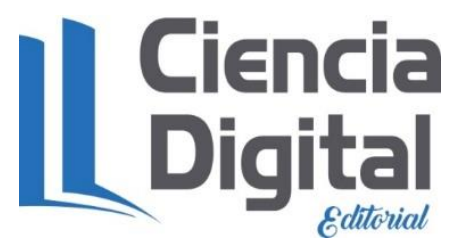

El artículo que se publica es de exclusiva responsabilidad de los autores y no necesariamente reflejan el pensamiento de la Revista Conciencia Digital.

El artículo queda en propiedad de la revista y, por tanto, su publicación parcial y/o total en otro medio tiene que ser autorizado por el director de la Revista Conciencia Digital.

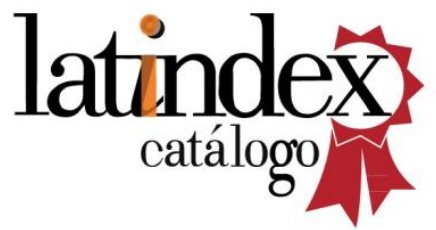

